\title{
Mathematical analysis of phase-field equations with numerically efficient coupling terms
}

\author{
MICHAL BENES̆ \\ Department of Mathematics, Faculty of Nuclear Sciences and Physical Engineering, Czech \\ Technical University of Prague, Trojanova 13, 12000 Prague, Czech Republic
}

[Received 22 November 1999 and in revised form 24 October 2000]

\begin{abstract}
This paper deals with the equations in a phase-field model with special terms coupling the heat equation and the equation of phase. A finer control of latent heat release together with a gradient coupling term in the phase equation are introduced as a consequence of an extensive numerical work with models of phase transitions within the context of the solidification of crystalline substances. We present a proof of the existence and uniqueness of the weak solution of the modified system of equations. Furthermore, we perform an asymptotic procedure to recover sharp-interface relations. Finally, several numerical studies demonstrate how the model behaves compared to its standard version.
\end{abstract}

Keywords: Phase field; microstructure growth; Stefan problem; compactness method

\section{Introduction}

The description of microscopic phenomena accompanying the solidification process of crystalline materials requires a simultaneous evaluation of the bulk enthalpy, the bulk and surface free energy of the system. A diffuse interpretation of the phase interface has its origin in the Cahn-Hilliard theory of solid-liquid phase transitions [9]. The equations known as the phase-field model have been analysed from the viewpoint of the existence and uniqueness of solution, in relation to the Stefan problem (see [7]), and convergence to the mean-curvature problem [6] in some special cases. The model based on phase-field equations exhibits satisfactory qualitative agreement with the real situation (see [3]). The model is able to demonstrate important microscopic phenomena appearing in the solidification of crystalline substances (dendritic or equiaxed growth, coarsening, ripening, reheating etc: see [3]). The question of quantitative agreement still remains open for the evolution of general, non-convex shapes like dendrites. The simulation of this phenomenon is limited by the power of currently used computers and requires more profound and sophisticated numerical algorithms to be used, but also opens discussion about the physical relevance of the phase-field model, or its particular parts. The behaviour of the equations in question can be investigated from the viewpoint of approximation of mean curvature, of influence of the diffusive interface on the release of latent heat and of stability of phase boundary. These points were discussed in [2] and led to modifications of coupling between the two equations proposed in view of better satisfaction of the sharp-interface relation-the Gibbs-Thompson equation. As the numerical behaviour of the model has been improved (for example see [5]), it is useful to analyse the equations in question from a mathematical point of view.

\footnotetext{
†Email: benes@km1.fjfi.cvut.cz
} 


\section{Equations}

We begin with the system of equations in [3] extensively used for the qualitative simulation of microstructure phenomena in solidification of crystallic materials:

$$
\begin{aligned}
\frac{\partial u}{\partial t} & =\Delta u+L \frac{\partial p}{\partial t}, \\
\alpha \xi^{2} \frac{\partial p}{\partial t} & =\xi^{2} \Delta p+f_{0}(p)+F(u) \xi^{2}|\nabla p|,
\end{aligned}
$$

with initial conditions

$$
\left.u\right|_{t=0}=u_{\text {ini }},\left.\quad p\right|_{t=0}=p_{\text {ini }},
$$

and with boundary conditions of the Dirichlet type

$$
\left.u\right|_{\partial \Omega}=0,\left.\quad p\right|_{\partial \Omega}=0 .
$$

Here, $\Omega$ is a bounded domain in $\mathbb{R}^{n}$ with a $C^{2}$ boundary (or alternatively, a convex domain with piecewise smooth boundary: see [12]), and $L, \alpha, \xi$ are positive constants.

The nonlinear function $f_{0}=-w_{0}^{\prime}$ is given by the derivative of a double-well potential $w_{0}$, which plays a key role in the model. The function $w_{0}$ is typically a polynomial of fourth degree with two (stable) minima at 0 and 1 , and one (unstable) maximum usually at 0.5 . The solution $p$ is expected to look for stable states 0 and 1 and to allow a relatively small space for values near 0.5 . We therefore identify the stable states with the liquid and solid phases, and the unstable state with a region between phases-a diffuse interface (see [7], [3]). The analysis below considers $f_{0}(p)=a p(1-p)\left(p-\frac{1}{2}\right)$ with $a>0$, but can be performed using any other function with the same properties.

The coupling function $F(u)$ is bounded and continuous, or even Lipschitz continuous, and is related to the undercooling of the interface (in fact, it is proportional to the undercooling of the interface in a certain range of values), and $|$.$| denotes the Euclidean norm in \mathbb{R}^{n}$. For the sake of simplicity, $n=2$ and the boundary conditions are homogeneous. Obviously, extension to higher dimensions and to other boundary conditions is possible.

In the physical context, the system (2.1) is treated as a regularization of the modified Stefan problem describing isotropic microstructure formation in solidification of a pure substance if $\xi \rightarrow 0$ (see [3], [7]):

$$
\begin{aligned}
& \frac{\partial u}{\partial t}=\Delta u \text { in } \Omega_{s} \text { and } \Omega_{l}, \\
& \left.u\right|_{\partial \Omega}=0,\left.\quad u\right|_{t=0}=u_{\mathrm{ini}}, \\
& \left.\frac{\partial u}{\partial n_{\Gamma}}\right|_{s}-\left.\frac{\partial u}{\partial n_{\Gamma}}\right|_{l}=L v_{\Gamma} \text { on } \Gamma(t), \\
& F(u)=\kappa_{\Gamma}+\alpha v_{\Gamma} \text { on } \Gamma(t), \\
& \left.\Omega_{S}(t)\right|_{t=0}=\Omega_{s, \mathrm{ini}},
\end{aligned}
$$

where $\Omega_{s}, \Omega_{l}$ are solid and liquid phases, respectively, and $u$ the temperature field. Discontinuity of heat flux on $\Gamma(t)$ is described by the Stefan condition (2.3), where $v_{\Gamma}$ is the velocity in the direction of the outer normal $\boldsymbol{n}_{\Gamma}$ to $\Omega_{s}$. The formula (2.4) is the Gibbs-Thompson relation on $\Gamma(t)$ whose mean curvature is denoted as $\kappa_{\Gamma}$. The parameter $\alpha$ is the coefficient of attachment kinetics. 


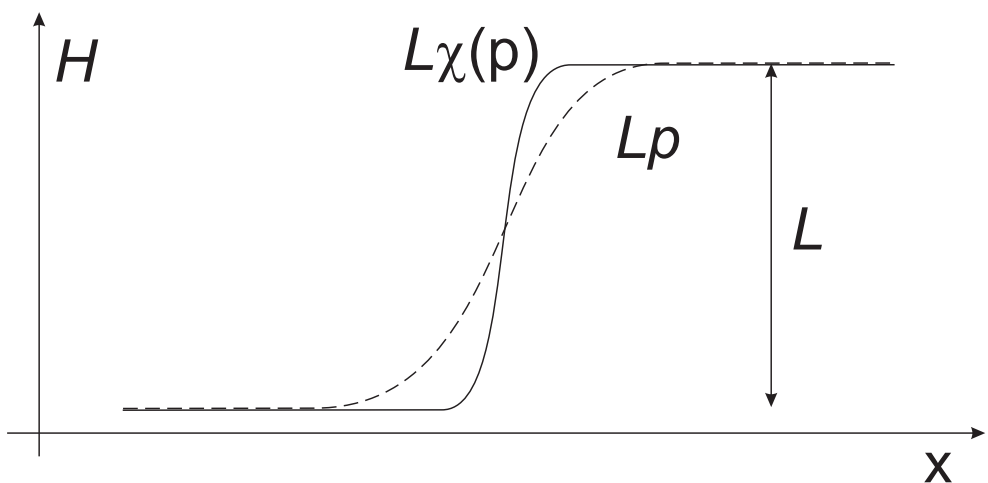

FIG. 1. Schematic illustration of the role of focusing latent heat.

Following [3], the relation between (2.1) and (2.2)-(2.4) can be studied with the use of asymptotic analysis.

The phase equation in (2.1) contains a modified coupling term $F(u) \xi^{2}|\nabla p|$, proposed in [2] as a consequence of the level-set reformulation of the condition (2.4) using the definition of the boundary $\Gamma$ as a manifold. The solution of (2.1), as is observed in numerical studies (see [3]), shows an insufficient convergence rate towards a sharp-interface limit in terms of the magnitude of $\xi$ required to obtain quantitatively reasonable results. Therefore, we propose a slight modification of the source term in the heat equation by a focusing function $\chi=\chi(p)$ satisfying $\chi(0)=0, \chi(1)=1, \chi(0.5)=$ 0.5 , whose derivative is bounded, Lipschitz continuous. The action of $\chi$ can be observed in Fig. 1 $(H(u, p)=u-L \chi(p))$. The paper presents an analysis for the phase-field equations including the above-mentioned improvement:

$$
\begin{aligned}
\frac{\partial u}{\partial t} & =\Delta u+L \chi^{\prime}(p) \frac{\partial p}{\partial t}, \\
\alpha \xi^{2} \frac{\partial p}{\partial t} & =\xi^{2} \Delta p+f_{0}(p)+F(u) \xi^{2}|\nabla p|,
\end{aligned}
$$

with the initial conditions

$$
\left.u\right|_{t=0}=u_{\text {ini }},\left.\quad p\right|_{t=0}=p_{\text {ini }},
$$

and with the boundary conditions of Dirichlet type

$$
\left.u\right|_{\partial \Omega}=0,\left.\quad p\right|_{\partial \Omega}=0
$$

Then, we relate model and physical parameters of the problem by using a matched asymptotic analysis. Equations (2.5) have (in comparison with those of [7]) no Lyapunov functional and are hard to be derived by minimization of an energy functional. On the other hand, the form of (2.5) is closely related to geometrical aspects of level-set motion by mean curvature [4]. 


\section{Existence and uniqueness}

First, we introduce the following notation:

$$
\begin{gathered}
(u, v)=\int_{\Omega} u(x) v(x) \mathrm{d} x, \quad\|u\|=\sqrt{\int_{\Omega} u(x)^{2} \mathrm{~d} x} \quad \text { for } \quad u, v \in \mathrm{L}_{2}(\Omega), \\
(\nabla u, \nabla v)=\int_{\Omega} \nabla u(x) \cdot \nabla v(x) \mathrm{d} x, \quad\|\nabla u\|=\sqrt{\int_{\Omega}|\nabla u(x)|^{2} \mathrm{~d} x} \quad \text { for } \quad u, v \in \mathrm{H}^{1}(\Omega) .
\end{gathered}
$$

We also note that the assumptions on $\chi$ imply that there are constants $C_{\chi}, L_{\chi}>0$ such that $\left|\chi^{\prime}(s)\right| \leqslant C_{\chi},\left|\chi^{\prime}\left(s_{1}\right)-\chi^{\prime}\left(s_{2}\right)\right| \leqslant L_{\chi}\left|s_{1}-s_{2}\right|$ for all $s, s_{1}, s_{2} \in \mathbb{R}$. Similarly, the assumptions on $F$ imply that there are constants $C_{F}, L_{F}>0$ such that $|F(s)| \leqslant C_{F}$, and $\left|F\left(s_{1}\right)-F\left(s_{2}\right)\right| \leqslant L_{F}\left|s_{1}-s_{2}\right|$ for all $s, s_{1}, s_{2} \in \mathbb{R}$. We define the notion of the weak solution as usual, as follows.

DEFINITION 3.1 The weak solution of the boundary-value problem with homogeneous Dirichlet boundary conditions for the phase-field equations (2.5) is a couple of functions $[u, p] \in$ $\mathrm{L}_{2}\left(0, T ;\left[\mathrm{H}_{0}^{1}(\Omega)\right]^{2}\right)$ such that it satisfies

$$
\begin{aligned}
\frac{\mathrm{d}}{\mathrm{d} t}(u-L \chi(p), v)+(\nabla u, \nabla v) & =0 \quad \text { a.e. in }(0, T), \\
\left.u\right|_{t=0} & =u_{\text {ini }}, \\
\alpha \xi^{2} \frac{\mathrm{d}}{\mathrm{d} t}(p, q)+\xi^{2}(\nabla p, \nabla q) & =\left(f_{0}(p), q\right)+\xi^{2}(F(u)|\nabla p|, q) \quad \text { a.e. in }(0, T), \\
\left.p\right|_{t=0} & =p_{\text {ini }},
\end{aligned}
$$

for each $v, q \in \mathrm{H}_{0}^{1}(\Omega)$.

The continuous embedding of $\mathrm{H}^{1}(\Omega)$ into $\mathrm{L}_{s}(\Omega)$ for each $s \in(1,+\infty)(\operatorname{dim} \Omega=2)$ ensures that $f_{0}(p) \in \mathrm{L}_{2}(\Omega)$ for almost all $t \in(0, T)$. If $[u, p] \in \mathrm{L}_{2}\left(0, T ;\left[\mathrm{H}_{0}^{1}(\Omega)\right]^{2}\right)$ solves $(3.1)$, then $[u, p]$ is a continuous mapping from the closed interval $\langle 0, T\rangle$ to $\left[\mathrm{H}^{-1}(\Omega)\right]^{2}$. Thus, the definition has the proper sense. Our existence and uniqueness result is contained in the following theorem. By its virtue, the proof investigates convergence of a semi-discrete scheme based on the Faedo-Galerkin method.

THEOREM 3.2 Consider the problem (3.1) in a bounded domain $\Omega \subset \mathbb{R}^{2}$ with a $C^{2}$ boundary, and with $F$ being a bounded continuous function, $\chi$ a function with $\chi(0)=0, \chi(1)=1, \chi(0.5)=0.5$, $\chi^{\prime}$ bounded, Lipschitz continuous. Assume that

$$
u_{\text {ini }}, p_{\text {ini }} \in \mathrm{H}^{1}(\Omega) .
$$

Then, there is a solution of the problem (3.1) satisfying

$$
\begin{gathered}
u, p \in \mathrm{L}_{\infty}\left(0, T ; \mathrm{H}_{0}^{1}(\Omega)\right), p \in \mathrm{L}_{2}\left(0, T ; \mathrm{H}^{2}(\Omega)\right), \\
\frac{\partial u}{\partial t}, \frac{\partial p}{\partial t} \in \mathrm{L}_{2}\left(0, T ; \mathrm{L}_{2}(\Omega)\right) .
\end{gathered}
$$

Additionally, if $F$ is Lipschitz continuous and $\chi(p)=p$, the solution is unique. 
Proof. We derive a sequence of approximate solutions to the original problem (3.1). Assume that there is an orthonormal basis $\left\{v_{i}\right\}_{i \in \mathbb{N}}$ of the Hilbert space $\mathrm{L}_{2}(\Omega)$ consisting of eigenvectors of the operator $-\Delta$ coupled with homogeneous Dirichlet boundary conditions. Additionally, we assume that $(\forall i \in \mathbb{N})\left(v_{i} \in \mathcal{C}^{2}(\Omega) \cap \mathcal{C}^{1}(\bar{\Omega})\right)$. Corresponding eigenvalues are denoted by $\left\{\lambda_{i}\right\}_{i \in \mathbb{N}}$. Let $V_{m}=$ $\operatorname{span}\left\{v_{i}\right\}_{i \in \mathbb{N}_{m}}$ be a finite-dimensional subspace $\left(\mathbb{N}_{m}=\{1, \ldots, m\}\right) ; \mathcal{P}_{m}: \mathrm{L}_{2}(\Omega) \rightarrow V_{m}$ be the $\mathrm{L}_{2}-$ projection operator (coinciding with the $\mathrm{H}^{1}$-projector). We seek for a solution $\left[u^{m}, p^{m}\right]$ from $\langle 0, T)$ to $\left[V_{m}\right]^{2}$ of an auxiliary problem:

$$
\begin{aligned}
\frac{\mathrm{d}}{\mathrm{d} t}\left(u^{m}-L \chi\left(p^{m}\right), v\right)+\left(\nabla u^{m}, \nabla v\right) & =0 \quad \text { a.e. in }(0, T), \forall v \in V_{m}, \\
u^{m}(0) & =\mathcal{P}_{m} u_{\text {ini }}, \\
\alpha \xi^{2} \frac{\mathrm{d}}{\mathrm{d} t}\left(p^{m}, q\right)+\xi^{2}\left(\nabla p^{m}, \nabla q\right)= & \left(f_{0}\left(p^{m}\right), q\right)+\xi^{2}\left(F\left(u^{m}\right)\left|\nabla p^{m}\right|, q\right) \\
& \text { a.e. in }(0, T), \forall q \in V_{m}, \\
p^{m}(0)= & \mathcal{P}_{m} p_{\text {ini }} .
\end{aligned}
$$

We use basic functions of $V_{m}$ to express the solution of (3.3) as

$$
u^{m}(t)=\sum_{i \in \mathbb{N}_{m}} \beta_{i}^{m}(t) v_{i}, \quad p^{m}(t)=\sum_{i \in \mathbb{N}_{m}} \gamma_{i}^{m}(t) v_{i},
$$

and to obtain a system of ordinary differential equations for the unknown functions of time: $\beta_{i}^{m}$, $\gamma_{i}^{m}$ :

$$
\begin{aligned}
\frac{\mathrm{d} \beta_{j}^{m}}{\mathrm{~d} t}+\lambda_{j} \beta_{j}^{m}= & L \frac{\mathrm{d}}{\mathrm{d} t}\left(\chi\left(\sum_{i \in \mathbb{N}_{m}} \gamma_{i}^{m} v_{i}\right), v_{j}\right) \quad \text { in }(0, T), \\
\beta_{j}^{m}(0)= & \beta_{j}^{0}, \quad \text { for each } \quad j \in \mathbb{N}_{m}, \\
\alpha \xi^{2} \frac{\mathrm{d} \gamma_{j}^{m}}{\mathrm{~d} t}+\xi^{2} \lambda_{j} \gamma_{j}^{m}= & \left(f_{0}\left(\sum_{i \in \mathbb{N}_{m}} \gamma_{i}^{m} v_{i}\right), v_{j}\right) \\
& +\xi^{2}\left(F\left(\sum_{i \in \mathbb{N}_{m}} \beta_{i}^{m} v_{i}\right)\left|\sum_{i \in \mathbb{N}_{m}} \gamma_{i}^{m} \nabla v_{i}\right|, v_{j}\right) \text { in }(0, T), \\
\gamma_{j}^{m}(0)= & \gamma_{j}^{0}, \quad \text { for each } \quad j \in \mathbb{N}_{m},
\end{aligned}
$$

where $\mathcal{P}_{m} u_{\text {ini }}=\sum_{i \in \mathbb{N}_{m}} \beta_{i}^{0} v_{i}, \quad \mathcal{P}_{m} p_{\text {ini }}=\sum_{i \in \mathbb{N}_{m}} \gamma_{i}^{0} v_{i}$. We follow the steps of the compactness method (see, for example, [18]), show that the solution of (3.4), (3.5) is defined on $(0, T)$ for $T>0$ and show an appropriate convergence of the couple $\left[u^{m}, p^{m}\right]$. For this purpose, we prove an a priori estimate by multiplying (3.4) by $\frac{\mathrm{d} \beta_{j}^{m}}{\mathrm{~d} t}$, (3.5) by $\frac{\mathrm{d} \gamma_{j}^{m}}{\mathrm{~d} t}$, and summing for $j \in \mathbb{N}_{m}$ :

$$
\begin{gathered}
\left\|\frac{\partial u^{m}}{\partial t}\right\|^{2}+\frac{1}{2} \frac{\mathrm{d}}{\mathrm{d} t}\left\|\nabla u^{m}\right\|^{2}=L\left(\frac{\partial \chi\left(p^{m}\right)}{\partial t}, \frac{\partial u^{m}}{\partial t}\right), \\
\alpha \xi^{2}\left\|\frac{\partial p^{m}}{\partial t}\right\|^{2}+\frac{\xi^{2}}{2} \frac{\mathrm{d}}{\mathrm{d} t}\left\|\nabla p^{m}\right\|^{2}=\left(f_{0}\left(p^{m}\right), \frac{\partial p^{m}}{\partial t}\right)+\xi^{2}\left(F\left(u^{m}\right)\left|\nabla p^{m}\right|, \frac{\partial p^{m}}{\partial t}\right) .
\end{gathered}
$$


Using the Schwarz and Young inequalities, we get

$$
\begin{array}{r}
\left\|\frac{\partial u^{m}}{\partial t}\right\|^{2}+\frac{\mathrm{d}}{\mathrm{d} t}\left\|\nabla u^{m}\right\|^{2} \leqslant L^{2} C_{\chi}^{2}\left\|\frac{\partial p^{m}}{\partial t}\right\|^{2}, \\
\frac{1}{2} \alpha \xi^{2}\left\|\frac{\partial p^{m}}{\partial t}\right\|^{2}+\frac{\xi^{2}}{2} \frac{\mathrm{d}}{\mathrm{d} t}\left\|\nabla p^{m}\right\|^{2}+\frac{\mathrm{d}}{\mathrm{d} t}\left(w_{0}\left(p^{m}\right), 1\right) \leqslant \frac{C_{F}^{2}}{2 \alpha} \xi^{2}\left\|\nabla p^{m}\right\|^{2},
\end{array}
$$

where $w_{0}^{\prime}=-f_{0}$. Combining these estimates, we have

$$
\begin{aligned}
& \frac{1}{4} \alpha \xi^{2}\left\|\frac{\partial p^{m}}{\partial t}\right\|^{2}+\frac{\alpha \xi^{2}}{4 L^{2} C_{\chi}^{2}}\left\|\frac{\partial u^{m}}{\partial t}\right\|^{2}+\frac{\alpha \xi^{2}}{4 L^{2} C_{\chi}^{2}} \frac{\mathrm{d}}{\mathrm{d} t}\left\|\nabla u^{m}\right\|^{2}+\frac{\xi^{2}}{2} \frac{\mathrm{d}}{\mathrm{d} t}\left\|\nabla p^{m}\right\|^{2} \\
& +\frac{\mathrm{d}}{\mathrm{d} t}\left(w_{0}\left(p^{m}\right), 1\right) \leqslant \frac{C_{F}^{2}}{2 \alpha} \xi^{2}\left\|\nabla p^{m}\right\|^{2} .
\end{aligned}
$$

Adding non-negative terms on the right-hand side,

$$
\begin{aligned}
& \frac{1}{4} \alpha \xi^{2}\left\|\frac{\partial p^{m}}{\partial t}\right\|^{2}+\frac{\alpha \xi^{2}}{4 L^{2} C_{\chi}^{2}}\left\|\frac{\partial u^{m}}{\partial t}\right\|^{2}+\frac{\alpha \xi^{2}}{4 L^{2} C_{\chi}^{2}} \frac{\mathrm{d}}{\mathrm{d} t}\left\|\nabla u^{m}\right\|^{2}+\frac{\xi^{2}}{2} \frac{\mathrm{d}}{\mathrm{d} t}\left\|\nabla p^{m}\right\|^{2}+\frac{\mathrm{d}}{\mathrm{d} t}\left(w_{0}\left(p^{m}\right), 1\right) \\
& \leqslant \frac{C_{F}^{2}}{\alpha}\left(\frac{\xi^{2}}{2}\left\|\nabla p^{m}\right\|^{2}+\frac{\alpha \xi^{2}}{4 L^{2} C_{\chi}^{2}}\left\|\nabla u^{m}\right\|^{2}+\left(w_{0}\left(p^{m}\right), 1\right)\right) .
\end{aligned}
$$

Integrating over $(0, t)$, we have

$$
\begin{aligned}
& \left(\frac{\alpha \xi^{2}}{4 L^{2} C_{\chi}^{2}}\left\|\nabla u^{m}\right\|^{2}+\frac{\xi^{2}}{2}\left\|\nabla p^{m}\right\|^{2}+\left(w_{0}\left(p^{m}\right), 1\right)\right)(t) \\
& \quad \leqslant\left(\frac{\alpha \xi^{2}}{4 L^{2} C_{\chi}^{2}}\left\|\nabla u^{m}\right\|^{2}+\frac{\xi^{2}}{2}\left\|\nabla p^{m}\right\|^{2}+\left(w_{0}\left(p^{m}\right), 1\right)\right)(0) \exp \left(\frac{C_{F}^{2}}{\alpha} t\right) .
\end{aligned}
$$

The assumption (3.2) of the theorem together with the coincidence of projectors in $\mathrm{L}_{2}$ and $\mathrm{H}^{1}$ imply that $\nabla \mathcal{P}_{m} p_{\text {ini }}, \nabla \mathcal{P}_{m} u_{\text {ini }}$ remain bounded in $\mathrm{L}_{2}(\Omega)$ uniformly in $m$, and $\mathcal{P}_{m} p_{\text {ini }}$ are bounded in $\mathrm{L}_{4}(\Omega)$ uniformly in $m$ (due to the continuous imbedding of $\mathrm{H}^{1}$ into $\mathrm{L}_{4}$ ). Consequently, the inequality (3.10) implies that, independent of $m, \nabla u^{m}, \nabla p^{m}$ are bounded in $\mathrm{L}_{\infty}\left(0, T ; \mathrm{L}_{2}(\Omega)\right)$, and $p^{m}$ are bounded in $\mathrm{L}_{\infty}\left(0, T ; \mathrm{L}_{4}(\Omega)\right)$ for each finite time $T>0$. This result is valid for any spatial dimension. The additional assumption $n=2$ together with the above-mentioned boundedness of the sequence $\nabla p^{m}$ implies through the embedding theorem that, independent of $m, p^{m}$ are bounded in $\mathrm{L}_{\infty}\left(0, T ; \mathrm{L}_{6}(\Omega)\right)$ for each finite time $T>0$, which can be used to strenghten the convergence of $f_{0}\left(p^{m}\right)$ as shown below.

Integrating (3.10) over $(0, T)$, we get

$$
\begin{aligned}
& \int_{0}^{T}\left(\frac{\alpha \xi^{2}}{4 L^{2} C_{\chi}^{2}}\left\|\nabla u^{m}\right\|^{2}+\frac{\xi^{2}}{2}\left\|\nabla p^{m}\right\|^{2}+\left(w_{0}\left(p^{m}\right), 1\right)\right)(t) \mathrm{d} t \\
& \quad \leqslant\left(\frac{\alpha \xi^{2}}{4 L^{2} C_{\chi}^{2}}\left\|\nabla u^{m}\right\|^{2}+\frac{\xi^{2}}{2}\left\|\nabla p^{m}\right\|^{2}+\left(w_{0}\left(p^{m}\right), 1\right)\right)(0) \frac{\alpha}{C_{F}^{2}}\left(\exp \left(\frac{C_{F}^{2}}{\alpha} T\right)-1\right) .
\end{aligned}
$$


We use this estimate for the integration of the relation (3.9), and we see that

$$
\begin{aligned}
\int_{0}^{T} & \left(\frac{1}{4} \alpha \xi^{2}\left\|\frac{\partial p^{m}}{\partial t}\right\|^{2}+\frac{\alpha \xi^{2}}{4 L^{2} C_{\chi}^{2}}\left\|\frac{\partial u^{m}}{\partial t}\right\|^{2}\right)(t) \mathrm{d} t \\
& +\left(\frac{\xi^{2}}{2}\left\|\nabla p^{m}\right\|^{2}+\frac{\alpha \xi^{2}}{4 L^{2} C_{\chi}^{2}}\left\|\nabla u^{m}\right\|^{2}+\left(w_{0}\left(p^{m}\right), 1\right)\right)(T) \\
\leqslant & \left(\frac{\xi^{2}}{2}\left\|\nabla p^{m}\right\|^{2}+\frac{\alpha \xi^{2}}{4 L^{2} C_{\chi}^{2}}\left\|\nabla u^{m}\right\|^{2}+\left(w_{0}\left(p^{m}\right), 1\right)\right)(0) \\
& +\frac{C_{F}^{2}}{\alpha} \int_{0}^{T}\left(\frac{\xi^{2}}{2}\left\|\nabla p^{m}\right\|^{2}+\frac{\alpha \xi^{2}}{4 L^{2} C_{\chi}^{2}}\left\|\nabla u^{m}\right\|^{2}+\left(w_{0}\left(p^{m}\right), 1\right)\right)(t) \mathrm{d} t \\
\leqslant & \left(\frac{\alpha \xi^{2}}{4 L^{2} C_{\chi}^{2}}\left\|\nabla u^{m}\right\|^{2}+\frac{\xi^{2}}{2}\left\|\nabla p^{m}\right\|^{2}+\left(w_{0}\left(p^{m}\right), 1\right)\right)(0) \exp \left(\frac{C_{F}^{2}}{\alpha} T\right) .
\end{aligned}
$$

Passing to a subsequence $m^{\prime}$, we have $u^{m^{\prime}} \rightarrow u$ and $p^{m^{\prime}} \rightarrow p$ in $\mathrm{L}_{2}\left(0, T ; \mathrm{H}_{0}^{1}(\Omega)\right)$. The nonlinear terms in (2.5) require a stronger convergence result. Using the compact-embedding theorem [17] with the setting

$$
\begin{aligned}
& \left\{u^{m}\right\}_{m=1}^{\infty} \text { bounded in } \mathrm{L}_{2}\left(0, T ; \mathrm{H}_{0}^{1}(\Omega)\right),\left\{\frac{\partial u^{m}}{\partial t}\right\}_{m=1}^{\infty} \text { bounded in } \mathrm{L}_{2}\left(0, T ; \mathrm{L}_{2}(\Omega)\right), \\
& \left\{p^{m}\right\}_{m=1}^{\infty} \text { bounded in } \mathrm{L}_{6}\left(0, T ; \mathrm{H}_{0}^{1}(\Omega)\right),\left\{\frac{\partial p^{m}}{\partial t}\right\}_{m=1}^{\infty} \text { bounded in } \mathrm{L}_{2}\left(0, T ; \mathrm{L}_{2}(\Omega)\right),
\end{aligned}
$$

we see that $\left\{u^{m^{\prime}}\right\}_{m^{\prime}=1}^{\infty}$ converges strongly in $\mathrm{L}_{2}\left(0, T ; \mathrm{L}_{2}(\Omega)\right)$, and $\left\{p^{m^{\prime}}\right\}_{m^{\prime}=1}^{\infty}$ converges strongly in $\mathrm{L}_{6}\left(0, T ; \mathrm{L}_{6}(\Omega)\right)$. The polynomial form of $f_{0}$ then implies the existence of the strong limit of $f_{0}\left(p^{m^{\prime}}\right)$ in $\mathrm{L}_{2}\left(0, T ; \mathrm{L}_{2}(\Omega)\right)$ being equal to $f_{0}(p)$. We also observe that the term $F\left(u^{m}\right)\left|\nabla p^{m}\right|$ is bounded in $\mathrm{L}_{2}\left(0, T ; \mathrm{L}_{2}(\Omega)\right)$, and, therefore, the subsequence converges weakly to a function $\tilde{F}$ in this space. Convergence of $\chi\left(p^{m}\right)$ in $\mathrm{L}_{2}\left(0, T ; \mathrm{L}_{2}(\Omega)\right)$ via subsequence is guaranteed by boundedness of $\chi^{\prime}$. Finally, the term $\chi^{\prime}\left(p^{m}\right) \frac{\partial p^{m}}{\partial t}$ is bounded in $\mathrm{L}_{2}\left(0, T ; \mathrm{L}_{2}(\Omega)\right)$ which implies the convergence of subsequence to a function $\tilde{\chi}$ in this space which is equal to $\frac{\partial \chi(p)}{\partial t}$ via definition of the time derivative in the sense of distributions. In order to be able to pass towards the limit in (3.3), we prove that $\tilde{F}=F(u)|\nabla p|$. For this purpose, we show more about the regularity of $p$.

LEMMA 3.3 Under the assumptions of the theorem, the function $p$ belongs to $\mathrm{L}_{2}\left(0, T ; \mathrm{H}_{0}^{1}(\Omega) \cap\right.$ $\left.\mathrm{H}^{2}(\Omega)\right)$.

Proof. Multiply equation (3.5) by $\left(q, v_{j}\right)$, for a $q \in \mathcal{D}(\Omega)$, and sum over $\mathbb{N}_{m}$ :

$$
\alpha \xi^{2}\left(\frac{\partial p^{m^{\prime}}}{\partial t}, \mathcal{P}_{m^{\prime}} q\right)+\xi^{2}\left(\nabla p^{m^{\prime}}, \nabla \mathcal{P}_{m^{\prime}} q\right)=\left(f_{0}\left(p^{m^{\prime}}\right), \mathcal{P}_{m^{\prime}} q\right)+\xi^{2}\left(F\left(u^{m^{\prime}}\right)\left|\nabla p^{m^{\prime}}\right|, \mathcal{P}_{m^{\prime}} q\right) .
$$

We can pass to the limit in the sense of $\mathcal{D}^{\prime}(0, T)$ by obtaining

$$
\alpha \xi^{2}\left(\frac{\partial p}{\partial t}, q\right)+\xi^{2}(\nabla p, \nabla q)=\left(f_{0}(p), q\right)+\xi^{2}(\tilde{F}, q) .
$$


Consequently, the function $p$ is continuous from $\langle 0, T\rangle$ into $\mathrm{L}_{2}(\Omega)$. We rewrite the previous equality in the sense of $\mathcal{D}^{\prime}(\Omega)$ :

$$
\alpha \xi^{2} \frac{\partial p}{\partial t}=\xi^{2} \Delta p+f_{0}(p)+\xi^{2} \tilde{F}
$$

As $\frac{\partial p}{\partial t}, f_{0}(p), \tilde{F}$ belong to $\mathrm{L}_{2}\left(0, T ; \mathrm{L}_{2}(\Omega)\right)$, it follows that $\Delta p \in \mathrm{L}_{2}\left(0, T ; \mathrm{L}_{2}(\Omega)\right)$. The continuity of the operator $\Delta^{-1}$ mapping $\mathrm{L}_{2}(\Omega)$ to $\mathrm{H}^{2}(\Omega) \cap \mathrm{H}_{0}^{1}(\Omega)$ —see [14]—implies that $p \in$ $\mathrm{L}_{2}\left(0, T ; \mathrm{H}^{2}(\Omega) \cap \mathrm{H}_{0}^{1}(\Omega)\right)$.

The next statement investigates the convergence of gradient.

LEMMA 3.4 The sequence $\nabla p^{m^{\prime}}$ converges strongly to $\nabla p$ in $\mathrm{L}_{2}\left(0, T ;\left[\mathrm{L}_{2}(\Omega)\right]^{2}\right)$.

Proof. The statement of the lemma is shown by following the technique used in [4]. Multiply equation (3.5) by $\gamma_{i}^{m}-\gamma_{i}$, where $p=\sum_{i \in \mathbb{N}} \gamma_{i} v_{i}$, sum over $i \in \mathbb{N}$ and integrate over $(0, T)$ :

$$
\begin{aligned}
& \alpha \xi^{2} \int_{0}^{T}\left(\frac{\partial p^{m^{\prime}}}{\partial t}, p^{m^{\prime}}-p\right) \mathrm{d} t+\xi^{2} \int_{0}^{T}\left(\nabla p^{m^{\prime}}, \nabla\left(p^{m^{\prime}}-p\right)\right) \mathrm{d} t \\
& \quad=\int_{0}^{T}\left(f_{0}\left(p^{m^{\prime}}\right), p^{m^{\prime}}-p\right) \mathrm{d} t+\xi^{2} \int_{0}^{T}\left(F\left(u^{m^{\prime}}\right)\left|\nabla p^{m^{\prime}}\right|, p^{m^{\prime}}-p\right) \mathrm{d} t .
\end{aligned}
$$

We add and subtract a term

$$
\xi^{2} \int_{0}^{T}\left(\nabla p, \nabla\left(p^{m^{\prime}}-p\right)\right) \mathrm{d} t
$$

to the equality (3.14) knowing that it tends to 0 as

$$
\nabla\left(p^{m^{\prime}}-p\right) \rightarrow 0
$$

weakly in $\mathrm{L}_{2}\left(0, T ;\left[\mathrm{L}_{2}(\Omega)\right]^{2}\right)$, and

$$
p^{m^{\prime}}-p \rightarrow 0
$$

strongly in $\mathrm{L}_{2}\left(0, T ; \mathrm{L}_{2}(\Omega)\right)$, if $m^{\prime} \rightarrow \infty$. Then, we have

$$
\begin{aligned}
\xi^{2} & \int_{0}^{T}\left(\nabla\left(p^{m^{\prime}}-p\right), \nabla\left(p^{m^{\prime}}-p\right)\right) \mathrm{d} t \\
= & -\alpha \xi^{2} \int_{0}^{T}\left(\frac{\partial p^{m^{\prime}}}{\partial t}, p^{m^{\prime}}-p\right) \mathrm{d} t+\int_{0}^{T}\left(f_{0}\left(p^{m^{\prime}}\right), p^{m^{\prime}}-p\right) \mathrm{d} t \\
& \quad+\xi^{2} \int_{0}^{T}\left(F\left(u^{m^{\prime}}\right)\left|\nabla p^{m^{\prime}}\right|, p^{m^{\prime}}-p\right) \mathrm{d} t+\xi^{2} \int_{0}^{T}\left(\nabla p, \nabla\left(p^{m^{\prime}}-p\right)\right) \mathrm{d} t .
\end{aligned}
$$

As all terms in the right-hand side tend to 0 if $m^{\prime} \rightarrow \infty$, we see that $\nabla\left(p^{m^{\prime}}-p\right) \rightarrow 0$ strongly in $\mathrm{L}_{2}\left(0, T ;\left[\mathrm{L}_{2}(\Omega)\right]^{2}\right)$, which gives the desired result.

LEMma 3.5 The sequence $F\left(u^{m^{\prime}}\right)\left|\nabla p^{m^{\prime}}\right|$ converges weakly to $F(u)|\nabla p|$ in $\mathrm{L}_{2}\left(0, T ; \mathrm{L}_{2}(\Omega)\right)$.

Proof. The sequence $\left|\nabla p^{m^{\prime}}\right|$ converges strongly in $\mathrm{L}_{2}\left(0, T ; \mathrm{L}_{2}(\Omega)\right)$ in the same way as is valid for $\nabla p^{m^{\prime}}$. The function $F$ is bounded and continuous, and $u^{m^{\prime}} \rightarrow u$ in $\mathrm{L}_{2}\left(0, T ; \mathrm{L}_{2}(\Omega)\right)$, then $F\left(u^{m^{\prime}}\right) \rightarrow F(u)$ strongly in $\mathrm{L}_{s}\left(0, T ; \mathrm{L}_{s}(\Omega)\right)$ for $1<s<+\infty$. This implies that the sequence $F\left(u^{m^{\prime}}\right)\left|\nabla p^{m^{\prime}}\right|$ converges strongly to $F(u)|\nabla p|$ in $\mathrm{L}_{\frac{2 s}{2+s}}\left(0, T ; \mathrm{L}_{\frac{2 s}{2+s}}(\Omega)\right)$. Then, the boundedness of the sequence $F\left(u^{m^{\prime}}\right)\left|\nabla p^{m^{\prime}}\right|$ in $\mathrm{L}_{2}\left(0, T ; \mathrm{L}_{2}(\Omega)\right)$ yields the statement of the lemma. 
3.0.1 Passage to the limit. Choose the test functions $w, q \in \mathcal{D}(\Omega)$, multiply (3.4) by ( $\left.w, v_{j}\right)$ and (3.5) by $\left(q, v_{j}\right)$, sum over $\mathbb{N}_{m}$. Then choose scalar functions $\varphi, \psi \in \mathcal{C}^{1}(\langle 0, T\rangle)$, for which $\varphi(T)=\psi(T)=0$. Integrate both equations by parts over $(0, T)$. Knowing that

(1) $\nabla p^{m^{\prime}}$ converges strongly in $\mathrm{L}_{2}\left(0, T ;\left[\mathrm{L}_{2}(\Omega)\right]^{2}\right)$ to $\nabla p$,

(2) $\mathcal{P}_{m^{\prime}} p_{\text {ini }}, \mathcal{P}_{m^{\prime}} u_{\text {ini }}$ converge strongly to $p_{\text {ini }}, u_{\text {ini }}$ in $\mathrm{L}_{2}(\Omega)$,

(3) $F\left(u^{m^{\prime}}\right)\left|\nabla p^{m^{\prime}}\right|$ converges weakly to $F(u)|\nabla p|$ in $\mathrm{L}_{2}\left(0, T ; \mathrm{L}_{2}(\Omega)\right)$,

(4) $\chi\left(p^{m^{\prime}}\right)$ converges strongly to $\chi(p)$ in $\mathrm{L}_{2}\left(0, T ; \mathrm{L}_{2}(\Omega)\right)$,

(5) $p^{m^{\prime}}(0)=\mathcal{P}_{m^{\prime}} p_{\text {ini }}, u^{m^{\prime}}(0)=\mathcal{P}_{m^{\prime}} u_{\text {ini }}$,

we are able to pass to the limit, and we obtain the following relations:

$$
\begin{aligned}
& \left(u_{\text {ini }}-L \chi\left(p_{\text {ini }}\right), w\right) \varphi(0)-\int_{0}^{T}(u-L \chi(p), w) \frac{\mathrm{d} \varphi}{\mathrm{d} t} \mathrm{~d} t+\int_{0}^{T} \varphi(\nabla u, \nabla w) \mathrm{d} t=0, \\
& \alpha \xi^{2}\left(p_{\text {ini }}, q\right) \psi(0)-\int_{0}^{T} \alpha \xi^{2}(p, q) \frac{\mathrm{d} \psi}{\mathrm{d} t} \mathrm{~d} t \\
& \quad+\int_{0}^{T} \psi\left[\xi^{2}(\nabla p, \nabla q)-\left(f_{0}(p), q\right)-\xi^{2}(F(u)|\nabla p|, q)\right] \mathrm{d} t=0 .
\end{aligned}
$$

If $\varphi, \psi \in \mathcal{D}(0, T)$, we have

$$
\begin{aligned}
\frac{\mathrm{d}}{\mathrm{d} t}(u-L \chi(p), w)+(\nabla u, \nabla w) & =0, \\
\alpha \xi^{2} \frac{\mathrm{d}}{\mathrm{d} t}(p, q)+\xi^{2}(\nabla p, \nabla q) & =\left(f_{0}(p), q\right)+\xi^{2}(F(u)|\nabla p|, q) .
\end{aligned}
$$

The weak solution satisfies the initial condition. Indeed, multiplying (3.16) by scalar functions $\varphi, \psi \in \mathcal{C}^{1}(\langle 0, T\rangle)$ for which $\varphi(T)=\psi(T)=0$ and integrating by parts over $(0, T)$ we obtain

$$
\begin{aligned}
& (u(0)-L \chi(p(0)), w) \varphi(0)-\int_{0}^{T}(u-L \chi(p), w) \frac{\mathrm{d} \varphi}{\mathrm{d} t} \mathrm{~d} t+\int_{0}^{T} \varphi(\nabla u, \nabla w) \mathrm{d} t=0 \\
& \alpha \xi^{2}(p(0), q) \psi(0)-\int_{0}^{T} \alpha \xi^{2}(p, q) \frac{\mathrm{d} \psi}{\mathrm{d} t} \mathrm{~d} t \\
& \quad+\int_{0}^{T} \psi\left[\xi^{2}(\nabla p, \nabla q)-\left(f_{0}(p), q\right)-\xi^{2}(F(u)|\nabla p|, q)\right] \mathrm{d} t=0 .
\end{aligned}
$$

Subtracting these equations from (3.15), we get

$\left(u_{\text {ini }}-L \chi\left(p_{\text {ini }}\right)-u(0)+L \chi(p(0)), w\right) \varphi(0)=0, \quad\left(p_{\text {ini }}-p(0), q\right) \psi(0)=0, \forall w, q \in \mathcal{D}(\Omega)$.

From this we see that $u(0)=u_{\text {ini }}, p(0)=p_{\text {ini }}$ in $\mathrm{L}_{2}(\Omega)$.

In the case when $F$ is Lipschitz continuous with the Lipschitz constant denoted by $L_{F}$ and when $\chi(p)=p$, we prove uniqueness of the solution of (3.1). We consider two solutions of the problem (3.1), denoted by $\left[u_{1}, p_{1}\right]$ and $\left[u_{2}, p_{2}\right]$. Subtracting corresponding systems of equations and denoting $\left[u_{12}, p_{12}\right]=\left[u_{1}-u_{2}, p_{1}-p_{2}\right]$, multiplying the first equation by $u_{12}-L p_{12}$ and the second equation by $p_{12}$, we have

$$
\begin{aligned}
\frac{1}{2} \frac{\mathrm{d}}{\mathrm{d} t}\left\|u_{12}-L p_{12}\right\|^{2}+\left\|\nabla\left(u_{12}-L p_{12}\right)\right\|^{2}+L\left(\nabla p_{12}, \nabla\left(u_{12}-L p_{12}\right)\right) & =0 \quad \text { in }(0, T) \\
\left(u_{12}-L p_{12}\right)(0) & =0
\end{aligned}
$$




$$
\begin{aligned}
\frac{1}{2} \alpha \xi^{2} \frac{\mathrm{d}}{\mathrm{d} t}\left\|p_{12}\right\|^{2}+\xi^{2}\left\|\nabla p_{12}\right\|^{2}= & \left(f_{0}\left(p_{1}\right)-f_{0}\left(p_{2}\right), p_{12}\right) \\
& +\xi^{2}\left(F\left(u_{1}\right)\left|\nabla p_{1}\right|-F\left(u_{2}\right)\left|\nabla p_{2}\right|, p_{12}\right) \text { in }(0, T), \\
p_{12}(0)= & 0 .
\end{aligned}
$$

Denote

$$
\Psi\left(p_{1}, p_{2}\right)=\frac{f_{0}\left(p_{1}\right)-f_{0}\left(p_{2}\right)}{p_{12}} .
$$

The a priori estimate (3.9) guarantees that there is a constant $C_{f}>0$ such that

$$
\left\|\Psi\left(p_{1}, p_{2}\right)\right\| \leqslant C_{f} \quad \text { in }(0, T)
$$

(as implied by the continuous embedding $\mathrm{H}_{0}^{1}(\Omega) \subset_{>} \mathrm{L}_{s}(\Omega)$ for $s \in\langle 1,+\infty)$ ). Therefore,

$$
\left|\left(\Psi\left(p_{1}, p_{2}\right) p_{12}, p_{12}\right)\right| \leqslant\left\|\Psi\left(p_{1}, p_{2}\right)\right\|\left\|p_{12}\right\|_{\mathrm{L}_{4}(\Omega)}^{2} \leqslant C_{f} C_{4}^{\prime}\left\|p_{12}\right\|\left\|\nabla p_{12}\right\|
$$

$\left(C_{4}^{\prime}>0\right.$, see [18]). Using the Poincaré, Young and Schwarz inequalities, we get

$$
\begin{gathered}
\frac{\mathrm{d}}{\mathrm{d} t}\left\|u_{12}-L p_{12}\right\|^{2}+\frac{1}{C_{\Omega}}\left\|u_{12}-L p_{12}\right\|^{2} \leqslant L^{2}\left\|\nabla p_{12}\right\|^{2}, \\
\frac{1}{2} \alpha \xi^{2} \frac{\mathrm{d}}{\mathrm{d} t}\left\|p_{12}\right\|^{2}+\xi^{2}\left\|\nabla p_{12}\right\|^{2} \leqslant C_{f} C_{4}^{\prime}\left\|p_{12}\right\|\left\|\nabla p_{12}\right\|+\xi^{2} L_{F}\left\|u_{12}\right\|\left\|\nabla p_{1}\right\|_{\mathrm{L}_{4}(\Omega)}\left\|p_{12}\right\|_{\mathrm{L}_{4}(\Omega)} \\
+\xi^{2} C_{F}\left\|\nabla p_{12}\right\|\left\|p_{12}\right\|,
\end{gathered}
$$

in $(0, T)$, where $C_{\Omega}$ appears in the Poincare inequality. Considering the fact that there is a constant $C_{p}$ for which

$$
\int_{0}^{T}\left\|\nabla p_{1}\right\|_{\mathrm{L}_{4}(\Omega)}^{2} \mathrm{~d} t \leqslant C_{4}^{2} \int_{0}^{T}\left\|p_{1}\right\|_{\mathrm{H}^{2}(\Omega)}^{2} \mathrm{~d} t \leqslant C_{p}^{2}
$$

where $C_{4}$ is the norm of the embedding $\mathrm{H}_{0}^{1}(\Omega)$ into $\mathrm{L}_{4}(\Omega)$, and $\Delta p_{1} \in \mathrm{L}_{2}\left(0, T ; \mathrm{L}_{2}(\Omega)\right)$, we obtain

$$
\begin{aligned}
\frac{\mathrm{d}}{\mathrm{d} t}\left\|u_{12}-L p_{12}\right\|^{2} & \leqslant L^{2}\left\|\nabla p_{12}\right\|^{2} \quad \text { in }(0, T) \\
\frac{1}{2} \alpha \xi^{2} \frac{\mathrm{d}}{\mathrm{d} t}\left\|p_{12}\right\|^{2}+\frac{\xi^{2}}{2}\left\|\nabla p_{12}\right\|^{2} \leqslant & \left(\xi^{-2}\left(C_{f} C_{4}^{\prime}+C_{F} \xi^{2}\right)^{2}+2 L^{2} \xi^{2} C_{4}^{2}\left\|\nabla p_{1}\right\|_{\mathrm{L}_{4}(\Omega)}^{2} L_{F}^{2}\right)\left\|p_{12}\right\|^{2} \\
& +2 C_{4}^{2}\left\|\nabla p_{1}\right\|_{\mathrm{L}_{4}(\Omega)}^{2} L_{F}^{2} \xi^{2}\left\|u_{12}-L p_{12}\right\|^{2} \quad \text { in }(0, T) .
\end{aligned}
$$

Combining these inequalities, we have in $(0, T)$ :

$$
\frac{\mathrm{d}}{\mathrm{d} t}\left(\frac{1}{2} \alpha \xi^{2}\left\|p_{12}\right\|^{2}+\frac{\xi^{2}}{2 L^{2}}\left\|u_{12}-L p_{12}\right\|^{2}\right) \leqslant M(t)\left(\frac{1}{2} \alpha \xi^{2}\left\|p_{12}\right\|^{2}+\frac{\xi^{2}}{2 L^{2}}\left\|u_{12}-L p_{12}\right\|^{2}\right)
$$

with

$$
M(t)=\frac{2\left(\xi^{-2}\left(C_{f} C_{4}^{\prime}+C_{F} \xi^{2}\right)^{2}+2 L^{2} \xi^{2} C_{4}^{2}\left\|\nabla p_{1}\right\|_{\mathrm{L}_{4}(\Omega)}^{2} L_{F}^{2}\right)}{\min (\alpha, 1) \xi^{2}} .
$$

Such an inequality, together with (3.17) and with the initial conditions, implies that

$$
p_{12}(t)=u_{12}(t)=0 \quad \text { in } \mathrm{L}_{2}(\Omega), \quad \forall t \in(0, T) .
$$

as follows from the Gronwall lemma. 


\section{Matched asymptotic analysis}

The relationship of the phase-field equations (2.5) to the sharp-interface problem (2.2)-(2.4) is demonstrated by the formal matched asymptotic analysis (the method is explained in [15]). A rigorous asymptotic procedure applied to the standard variant of phase-field equations has been performed in [7].

\subsection{Assumptions}

We assume that $\chi^{\prime \prime}$ is smooth, the solution of equations (2.5)

$$
u=u(t, \boldsymbol{x} ; \xi), \quad p=p(t, \boldsymbol{x} ; \xi),
$$

is sufficiently smooth, and $\nabla p$ never vanishes on the level-set $p=\frac{1}{2}$

$$
\left.\nabla p\right|_{p=\frac{1}{2}} \neq 0 \text {. }
$$

A very small diffusion term and derivative of a double-well function in the equation of phase lets appear a thin layer $\Omega_{\Gamma}$ between the two major domains (phases), where the function $p$ quickly changes its value (see [1]). To study such a behaviour in detail, the asymptotic analysis with respect to the 'small' parameter $\xi$ is applied. The formal procedure has been applied to the system containing the Allen-Cahn equation in [16], and here it is extended to equations (2.5).

DEFINITION 4.1 We define

$$
\begin{aligned}
\Omega_{S}(t ; \xi) & =\{\boldsymbol{x} \in \Omega \mid p(t, \boldsymbol{x} ; \xi)>0.5\}, \\
\Omega_{l}(t ; \xi) & =\{\boldsymbol{x} \in \Omega \mid p(t, \boldsymbol{x} ; \xi)<0.5\}, \\
\Gamma(t ; \xi) & =\{\boldsymbol{x} \in \Omega \mid p(t, \boldsymbol{x} ; \xi)=0.5\} .
\end{aligned}
$$

The set $\Omega_{s}$ is called solid domain, the set $\Omega_{l}$ is called liquid domain, and the set $\Gamma$ is called phase interface.

According to (4.1), any pathological behaviour like fattening (described in [10]) is excluded. The hypersurface $\Gamma$ can be parametrized with a sufficiently smooth mapping $\boldsymbol{Q}$

$$
\Gamma(t)=\left\{\boldsymbol{x} \in \Omega \mid\left(\exists_{1} s \in S\right)(\boldsymbol{x}=\boldsymbol{Q}(s))\right\},
$$

locally in $t$ where $S \subset \mathbb{R}, \boldsymbol{Q}: \mathrm{S} \rightarrow \Omega$ and

$$
(\forall s \in \mathrm{S})(p(t, \boldsymbol{Q}(s) ; \xi)=0.5) .
$$

This also means that $\boldsymbol{Q}=\boldsymbol{Q}(t, s ; \xi)$. Consequently, a local orthogonal system of coordinates can be introduced, in the neighbourhood of $\Gamma$

$$
\boldsymbol{x}=\boldsymbol{Q}(t, s ; \xi)+r \boldsymbol{n}_{\Gamma}(t, s ; \xi),
$$

where $s$ are longitudinal and $r$ radial (the signed distance function, see [16]) coordinates, and $\boldsymbol{n}_{\Gamma}$ is unit normal to $\Gamma$ pointing in the direction of decreasing $p$. The implicit-function theorem justifies the existence of an inverse mapping

$$
s=s(t, \boldsymbol{x} ; \xi), \quad r=r(t, \boldsymbol{x} ; \xi) .
$$

Previous definitions directly imply $\left(\boldsymbol{Q}=\left[Q_{1}, Q_{2}\right], \boldsymbol{n}_{\Gamma}=\left[n_{1}, n_{2}\right]\right)$ the following lemma. 
LEMMA 4.2 On $\Gamma(t)$, the following relations hold:

$$
\sum_{i=1}^{2} n_{i}^{2}(s)=1, \quad \sum_{i=1}^{2} n_{i}(s) \frac{\partial n_{i}(s)}{\partial s}=0, \quad \sum_{i=1}^{2} n_{i}(s) \frac{\partial Q_{i}(s)}{\partial s}=0 .
$$

The transformation of the differential operator $\nabla$ expressed in Euclidean coordinates (denoted by $\left.\nabla_{(x)}\right)$ to the local coordinates $(s, r)$ (denoted by $\left.\nabla_{(s, r)}\right)$ is given by

$$
\nabla_{(s, r)}=\mathcal{J} \cdot \nabla_{(x)},
$$

where

$$
\mathcal{J}=\left(\begin{array}{cc}
\frac{\partial Q_{1}}{\partial s}+r \frac{\partial n_{1}}{\partial s} & \frac{\partial Q_{2}}{\partial s}+r \frac{\partial n_{2}}{\partial s} \\
n_{1} & n_{2}
\end{array}\right)
$$

and

$$
\mathcal{J}^{-1}=\frac{1}{\operatorname{det} \mathcal{J}}\left(\begin{array}{cc}
n_{2} & -\frac{\partial Q_{2}}{\partial s}-r \frac{\partial n_{2}}{\partial s} \\
-n_{1} & \frac{\partial Q_{1}}{\partial s}+r \frac{\partial n_{1}}{\partial s}
\end{array}\right) .
$$

As a consequence, the Laplace operator expressed in the local coordinate system is

$$
\Delta_{(x)}=\frac{1}{\operatorname{det}^{2} \mathcal{J}} \frac{\partial^{2}}{\partial s^{2}}+\frac{\partial^{2}}{\partial r^{2}}+\frac{1}{\operatorname{det} \mathcal{J}} \frac{\partial}{\partial r}(\operatorname{det} \mathcal{J}) \frac{\partial}{\partial r}+\frac{1}{\operatorname{det} \mathcal{J}} \frac{\partial}{\partial s}\left(\frac{1}{\operatorname{det} \mathcal{J}}\right) \frac{\partial}{\partial s} .
$$

The other quantities appearing in the analysis can be expressed as follows:

$$
\kappa_{\Gamma}=\Delta_{(x)} r=\frac{1}{\operatorname{det} \mathcal{J}} \frac{\partial}{\partial r}(\operatorname{det} \mathcal{J}), \quad\left|\nabla_{(x)} r\right|=1, \quad \nabla_{(x)} r=\boldsymbol{n}_{\Gamma} \quad \text { on } \Gamma(t) .
$$

For the normal velocity of $\Gamma(t)$ and the normal unit vector we obtain

$$
\boldsymbol{v}_{\Gamma}=\left[\frac{\mathrm{d} x_{1}}{\mathrm{~d} t}, \frac{\mathrm{d} x_{2}}{\mathrm{~d} t}\right], \quad \boldsymbol{n}_{\Gamma}=-\frac{\nabla_{(x)} p}{\left|\nabla_{(x)} p\right|}, \quad v_{\Gamma}=\boldsymbol{v}_{\Gamma} \cdot \boldsymbol{n}_{\Gamma}=\frac{1}{\left|\nabla_{(x)} p\right|} \frac{\partial p}{\partial t} .
$$

This corresponds to the convention that the normal vector points out of $\Omega_{S}$, and $\kappa_{\Gamma}>0$ for $\Omega_{S}$ convex. We investigate the functions $[u, p]$ on $\Omega_{s}(t ; \xi), \Omega_{l}(t ; \xi)$ (the outer expansion), and in a close neighbourhood of $\Gamma(t)$ denoted by $\Omega_{\Gamma}(t ; \xi)$ (the inner expansion) separately. For this purpose, we assume that the functions $\boldsymbol{Q}, \boldsymbol{n}_{\Gamma}, v_{\Gamma}, \kappa_{\Gamma}$ (i.e. the sum of principal curvatures) and $\hat{\kappa}_{\Gamma}$ (i.e. the sum of squares of principal curvatures) admit the following expansions in terms of $\xi$ for $s \in S$ and $t \in\langle 0, T\rangle$ :

$$
\begin{aligned}
\boldsymbol{Q} & =\boldsymbol{Q}_{0}+\xi \boldsymbol{Q}_{1}+\xi^{2} \boldsymbol{Q}_{2}+\mathcal{O}\left(\xi^{3}\right), \\
\boldsymbol{n}_{\Gamma} & =\boldsymbol{n}_{\Gamma, 0}+\xi \boldsymbol{n}_{\Gamma, 1}+\mathcal{O}\left(\xi^{2}\right), \\
v_{\Gamma} & =v_{\Gamma, 0}+\xi v_{\Gamma, 1}+\mathcal{O}\left(\xi^{2}\right), \\
\kappa_{\Gamma} & =\kappa_{\Gamma, 0}+\xi \kappa_{\Gamma, 1}+\mathcal{O}\left(\xi^{2}\right), \\
\hat{\kappa}_{\Gamma} & =\hat{\kappa}_{\Gamma, 0}+\mathcal{O}(\xi) .
\end{aligned}
$$

For $t \in\langle 0, T\rangle$, the mapping $Q_{0}(\cdot, t): S \rightarrow \mathbb{R}^{2}$ parametrizes a closed differentiable simple curve $\Gamma_{0}(t)$ (see [13]), whose normal vector is $\boldsymbol{n}_{\Gamma, 0}$, normal velocity $v_{\Gamma, 0}$, and curvatures $\kappa_{\Gamma, 0}, \hat{\kappa}_{\Gamma, 0}$. We investigate the relation of $\Gamma(t)$ and $\Gamma_{0}(t)$. 


\subsection{Initial interface}

We assume that the interface $\Gamma$ is present since the initial moment $(\Gamma(0)=\Sigma)$, when it does not exhibit any dependence on $\xi$ :

$$
\Sigma=\left\{\boldsymbol{x} \in \mathbb{R}^{2} \mid \boldsymbol{x}=\boldsymbol{Q}_{\Sigma}(s), s \in S\right\} .
$$

This will imply the following equalities (for all $s \in S$ and $\xi>0$ ):

$$
\begin{aligned}
\boldsymbol{Q}_{0}(s, 0) & =\boldsymbol{Q}_{\Sigma}(s), & & \boldsymbol{Q}_{1}(s, 0)=\boldsymbol{Q}_{2}(s, 0)=0, \\
\boldsymbol{n}_{\Gamma, 0}(s, 0) & =\boldsymbol{n}_{\Sigma}(s), & & \boldsymbol{n}_{\Gamma, 1}(s, 0)=0, \\
\kappa_{\Gamma, 0}(s, 0) & =\kappa_{\Sigma}(s), & & \kappa_{\Gamma, 1}(s, 0)=0 .
\end{aligned}
$$

\subsection{Outer expansion}

Assume the asymptotic expansions of $u$ and $p$ in the following form:

$$
\begin{aligned}
& u(t, \boldsymbol{x} ; \xi)=u_{0}(t, \boldsymbol{x})+u_{1}(t, \boldsymbol{x}) \xi+u_{2}(t, \boldsymbol{x}) \xi^{2}+\mathcal{O}\left(\xi^{3}\right), \\
& p(t, \boldsymbol{x} ; \xi)=p_{0}(t, \boldsymbol{x})+p_{1}(t, \boldsymbol{x}) \xi+p_{2}(t, \boldsymbol{x}) \xi^{2}+\mathcal{O}\left(\xi^{3}\right),
\end{aligned}
$$

locally in $t$. Substituting such expansions into (2.5) and comparing terms at corresponding powers of $\xi$ up to the order 2, we get the following lemma.

LEMMA 4.3 The following outer relations hold:

$\mathcal{O}(1)$ :

$$
\begin{aligned}
\frac{\partial u_{0}}{\partial t} & =\Delta u_{0}+L \chi^{\prime}\left(p_{0}\right) \frac{\partial p_{0}}{\partial t}, \\
0 & =f_{0}\left(p_{0}\right) .
\end{aligned}
$$

$\mathcal{O}(\xi)$

$$
\begin{aligned}
\frac{\partial u_{1}}{\partial t} & =\Delta u_{1}+L\left(\chi^{\prime}\left(p_{0}\right) \frac{\partial p_{1}}{\partial t}+\chi^{\prime \prime}\left(p_{0}\right) p_{1} \frac{\partial p_{0}}{\partial t}\right), \\
0 & =f_{0}^{\prime}\left(p_{0}\right) p_{1} .
\end{aligned}
$$

$\mathcal{O}\left(\xi^{2}\right):$

$$
\begin{aligned}
\frac{\partial u_{2}}{\partial t}= & \Delta u_{2} \\
& +L\left(\chi^{\prime}\left(p_{0}\right) \frac{\partial p_{2}}{\partial t}+\chi^{\prime \prime}\left(p_{0}\right) p_{1} \frac{\partial p_{1}}{\partial t}+\chi^{\prime \prime}\left(p_{0}\right) p_{2} \frac{\partial p_{0}}{\partial t}+\frac{1}{2} \chi^{\prime \prime \prime}\left(p_{0}\right) p_{1}^{2} \frac{\partial p_{0}}{\partial t}\right) \\
\alpha \frac{\partial p_{0}}{\partial t}= & \Delta p_{0}+f_{0}^{\prime}\left(p_{0}\right) p_{2}+\frac{1}{2} f_{0}^{\prime \prime}\left(p_{0}\right) p_{1}^{2}+F\left(u_{0}\right)\left|\nabla p_{0}\right| .
\end{aligned}
$$

Relation (4.5) gives two stable minima $p_{0}=0,1$ corresponding to stable equilibrium states called solid and liquid (see Definition 4.1). Relations (4.7), (4.9) then yield $p_{1}=0, p_{2}=0$. The functions $u_{0,1,2}$ are solutions of the linear heat equations (4.4), (4.6), (4.8) with appropriate boundary and initial conditions in two separate domains $\Omega_{l, s}(t)$. 


\subsection{Inner expansion}

We proceed by the study of the solution within the transition layer near $\Gamma(t)$. We use the transform (4.2) with stretching

$$
r=\xi z
$$

to convert equations (2.5). Denote the functions

$$
\bar{u}(z, s, t ; \xi)=u(x, t ; \xi), \quad \bar{p}(z, s, t ; \xi)=p(x, t ; \xi) .
$$

The inner form of equations (2.5) is

$$
\begin{aligned}
& \frac{\partial \bar{u}}{\partial t}+\frac{\partial \bar{u}}{\partial s} \frac{\partial s}{\partial t}+\frac{\partial \bar{u}}{\partial z} \frac{\partial z}{\partial t}=\frac{1}{\xi^{2}} \frac{\partial^{2} \bar{u}}{\partial z^{2}}+\frac{1}{\operatorname{det}^{2} \mathcal{J}} \frac{\partial^{2} \bar{u}}{\partial s^{2}}+\frac{1}{\xi \operatorname{det} \mathcal{J}} \frac{\partial}{\partial r}(\operatorname{det} \mathcal{J}) \frac{\partial \bar{u}}{\partial z} \\
& +\frac{1}{\operatorname{det} \mathcal{J}} \frac{\partial}{\partial s}\left(\frac{1}{\operatorname{det} \mathcal{J}}\right) \frac{\partial \bar{u}}{\partial s}+L \chi^{\prime}(\bar{p})\left(\frac{\partial \bar{p}}{\partial t}+\frac{\partial \bar{p}}{\partial s} \frac{\partial s}{\partial t}+\frac{\partial \bar{p}}{\partial z} \frac{\partial z}{\partial t}\right), \\
& \alpha \xi^{2}\left(\frac{\partial \bar{p}}{\partial t}+\frac{\partial \bar{p}}{\partial s} \frac{\partial s}{\partial t}+\frac{\partial \bar{p}}{\partial z} \frac{\partial z}{\partial t}\right)=\xi^{2}\left(\frac{1}{\xi^{2}} \frac{\partial^{2} \bar{p}}{\partial z^{2}}+\frac{1}{\operatorname{det}^{2} \mathcal{J}} \frac{\partial^{2} \bar{p}}{\partial s^{2}}+\frac{1}{\xi \operatorname{det} \mathcal{J}} \frac{\partial}{\partial r}(\operatorname{det} \mathcal{J}) \frac{\partial \bar{p}}{\partial z}\right. \\
& \left.+\frac{1}{\operatorname{det} \mathcal{J}} \frac{\partial}{\partial s}\left(\frac{1}{\operatorname{det} \mathcal{J}}\right) \frac{\partial \bar{p}}{\partial s}\right)+f_{0}(\bar{p})+\xi^{2} F(\bar{u})\left|\mathcal{J}^{-1}\left(\begin{array}{c}
\frac{\partial \bar{p}}{\partial s} \\
\frac{1}{\xi} \frac{\partial \bar{p}}{\partial z}
\end{array}\right)\right| \text {. }
\end{aligned}
$$

The asymptotic expansion of the functions in question with respect to powers of $\xi$ is

$$
\begin{gathered}
\bar{u}(z, s, t ; \xi)=\bar{u}_{0}(z, s, t)+\bar{u}_{1}(z, s, t) \xi+\bar{u}_{2}(z, s, t) \xi^{2}+\mathcal{O}\left(\xi^{3}\right), \\
\bar{p}(z, s, t ; \xi)=\bar{p}_{0}(z, s, t)+\bar{p}_{1}(z, s, t) \xi+\bar{p}_{2}(z, s, t) \xi^{2}+\mathcal{O}\left(\xi^{3}\right), \\
r(z, s, t ; \xi)=r_{0}(z, s, t)+r_{1}(z, s, t) \xi+\mathcal{O}\left(\xi^{2}\right), \\
\frac{\partial r}{\partial t}=-v_{\Gamma, 0}-\xi v_{\Gamma, 1}+\mathcal{O}\left(\xi^{2}\right), \quad \nabla_{(x)} r=\boldsymbol{n}_{\Gamma, 0}+\xi \boldsymbol{n}_{\Gamma, 1}+\mathcal{O}\left(\xi^{2}\right), \\
\Delta_{(x)} r=\kappa_{\Gamma}+r \hat{\kappa}_{\Gamma}+\mathcal{O}\left(r^{2}\right)=\kappa_{\Gamma, 0}+\xi\left(\kappa_{\Gamma, 1}+z \hat{\kappa}_{\Gamma, 0}\right)+\mathcal{O}\left(\xi^{2}\right),
\end{gathered}
$$

locally in $t$ (also see [11]). Substituting previous expansions into (4.10), (4.11) and extracting relations of coefficients at corresponding powers of $\xi$, we get the following lemma.

LEMMA 4.4 Order equalities of the inner expansion:

$\mathcal{O}(1)$ :

$$
\begin{aligned}
\frac{\partial^{2} \bar{u}_{0}}{\partial z^{2}} & =0, \\
\frac{\partial^{2} \bar{p}_{0}}{\partial z^{2}}+f_{0}\left(\bar{p}_{0}\right) & =0 .
\end{aligned}
$$

$\mathcal{O}(\xi)$

$$
\begin{aligned}
-\frac{\partial \bar{u}_{0}}{\partial z} v_{\Gamma, 0} & =\frac{\partial^{2} \bar{u}_{1}}{\partial z^{2}}+\kappa_{\Gamma, 0} \frac{\partial \bar{u}_{0}}{\partial z}-L \chi^{\prime}\left(\bar{p}_{0}\right) \frac{\partial \bar{p}_{0}}{\partial z} v_{\Gamma, 0}, \\
-\alpha \frac{\partial \bar{p}_{0}}{\partial z} v_{\Gamma, 0} & =\frac{\partial^{2} \bar{p}_{1}}{\partial z^{2}}+\kappa_{\Gamma, 0} \frac{\partial \bar{p}_{0}}{\partial z}+F\left(\bar{u}_{0}\right)\left|\frac{\partial \bar{p}_{0}}{\partial z}\right|+f_{0}^{\prime}\left(\bar{p}_{0}\right) \bar{p}_{1} .
\end{aligned}
$$


$\mathcal{O}\left(\xi^{2}\right):$

$$
\begin{aligned}
& \frac{\partial \bar{u}_{0}}{\partial t}+\frac{\partial \bar{u}_{0}}{\partial s} \frac{\partial s_{0}}{\partial t}-\frac{\partial \bar{u}_{0}}{\partial z} v_{\Gamma, 1}-\frac{\partial \bar{u}_{1}}{\partial z} v_{\Gamma, 0}=\frac{\partial^{2} \bar{u}_{2}}{\partial z^{2}}+\frac{1}{\operatorname{det}^{2} \mathcal{J}_{0}} \frac{\partial^{2} \bar{u}_{0}}{\partial s^{2}} \\
& \quad+\kappa_{\Gamma, 0} \frac{\partial \bar{u}_{1}}{\partial z}+\left(\kappa_{\Gamma, 1}+z \kappa_{s, 0}\right) \frac{\bar{u}_{0}}{\partial z}+\left.\frac{1}{\operatorname{det} \mathcal{J}} \frac{\partial}{\partial s}(\operatorname{det} \mathcal{J})\right|_{0} \frac{\partial \bar{u}_{0}}{\partial s} \\
& \quad+L \chi^{\prime}\left(\bar{p}_{0}\right)\left(\frac{\partial \bar{p}_{0}}{\partial t}+\frac{\partial \bar{p}_{0}}{\partial s} \frac{\partial s_{0}}{\partial t}-\frac{\partial \bar{p}_{0}}{\partial z} v_{\Gamma, 1}-\frac{\partial \bar{p}_{1}}{\partial z} v_{\Gamma, 0}\right)-L \chi^{\prime \prime}\left(\bar{p}_{0}\right) \bar{p}_{1} v_{\Gamma, 0} \frac{\partial \bar{p}_{0}}{\partial z}, \\
& \alpha\left(\frac{\partial \bar{p}_{0}}{\partial t}-\frac{\partial \bar{p}_{0}}{\partial z} v_{\Gamma, 1}-\frac{\partial \bar{p}_{1}}{\partial z} v_{\Gamma, 0}\right)=\frac{\partial^{2} \bar{p}_{2}}{\partial z^{2}}+\kappa_{\Gamma, 0} \frac{\partial \bar{p}_{1}}{\partial z}+\left(\kappa_{\Gamma, 1}+z \kappa_{s, 0}\right) \frac{\bar{p}_{0}}{\partial z} \\
& \quad+f_{0}^{\prime}\left(\bar{p}_{0}\right) \bar{p}_{2}+\frac{1}{2} f^{\prime \prime}\left(\bar{p}_{0}\right) \bar{p}_{1}^{2}+\left|\frac{\partial \bar{p}_{0}}{\partial z}\right| F^{\prime}\left(\bar{u}_{0}\right) \bar{u}_{1}+F\left(\bar{u}_{0}\right) \frac{\frac{\partial \bar{p}_{0}}{\partial z}}{\left|\frac{\partial \bar{p}_{0}}{\partial z}\right|} \frac{\partial \bar{p}_{1}}{\partial z} .
\end{aligned}
$$

Relation (4.13) for $z \in \mathbb{R}$ has the only solution bounded in $\mathbb{R}$ in the form $\bar{u}_{0}=$ const. We multiply (4.14) by $\frac{\partial \bar{p}_{0}}{\partial z}$ and integrate over $(-\infty, z)$ with zero far-field condition $($ at $+\infty)$ for both $\bar{p}_{0}$ and $\frac{\partial \bar{p}_{0}}{\partial z}$ :

$$
\frac{1}{2}\left(\frac{\partial \bar{p}_{0}}{\partial z}\right)^{2}-w_{0}\left(\bar{p}_{0}\right)=0 .
$$

The sign convention for solid then gives

$$
\int_{0}^{z} \frac{\frac{\partial \bar{p}_{0}}{\partial z}}{\sqrt{2 w_{0}\left(\bar{p}_{0}\right)}} \mathrm{d} z=-z
$$

from which the function $\bar{p}_{0}$ is obtained as the inverse of an elliptic function with the far-field conditions

$$
\lim _{z \rightarrow-\infty} \bar{p}_{0}=1, \quad \lim _{z \rightarrow+\infty} \bar{p}_{0}=0 .
$$

The main interface relations are given as follows.

THEOREM 4.5 On the manifold $\Gamma_{0}$, the Stefan condition for the absolute terms in the outer expansion of temperature holds:

$$
\left.\frac{\partial u_{0}}{\partial r}\right|_{s}-\left.\frac{\partial u_{0}}{\partial r}\right|_{l}=L v_{\Gamma, 0}
$$

and the Gibbs-Thompson law for the absolute term in the inner expansion of the phase function holds:

$$
\int_{\mathbb{R}}\left(-\kappa_{\Gamma, 0} \frac{\partial \bar{p}_{0}}{\partial z}-F\left(\bar{u}_{0}\right)\left|\frac{\partial \bar{p}_{0}}{\partial z}\right|-\alpha \frac{\partial \bar{p}_{0}}{\partial z} v_{\Gamma, 0}\right) \frac{\partial \bar{p}_{0}}{\partial z} \mathrm{~d} z=0 .
$$

Proof. Relation (4.15) in the form

$$
0=\frac{\partial^{2} \bar{u}_{1}}{\partial z^{2}}-L \chi^{\prime}\left(\bar{p}_{0}\right) \frac{\partial \bar{p}_{0}}{\partial z} v_{\Gamma, 0}
$$


yields the function $\bar{u}_{1}$. It can be integrated, which gives

$$
\frac{\partial \bar{u}_{1}}{\partial z}-L \chi\left(\bar{p}_{0}\right) v_{\Gamma, 0}=c_{1}
$$

The matching condition at the interface $\Gamma$ from below and above (according to [8])

$$
\lim _{z \rightarrow \pm \infty} \frac{\partial \bar{u}_{1}}{\partial z}=\lim _{r \rightarrow \pm 0} \frac{\partial u_{0}}{\partial r}
$$

and properties of $\chi$

$$
\lim _{z \rightarrow+\infty} \chi\left(\bar{p}_{0}\right)=\chi(0)=0, \quad \lim _{z \rightarrow-\infty} \chi\left(\bar{p}_{0}\right)=\chi(1)=1
$$

lead to subtracting the upper and lower limit at $\Gamma_{0}$, and to recovering the Stefan condition:

$$
\left.\frac{\partial u_{0}}{\partial r}\right|_{s}-\left.\frac{\partial u_{0}}{\partial r}\right|_{l}=L v_{\Gamma, 0}
$$

The relation (4.16) has the form

$$
\frac{\partial^{2} \bar{p}_{1}}{\partial z^{2}}+f_{0}^{\prime}\left(\bar{p}_{0}\right) \bar{p}_{1}=-\kappa_{\Gamma, 0} \frac{\partial \bar{p}_{0}}{\partial z}-F\left(\bar{u}_{0}\right)\left|\frac{\partial \bar{p}_{0}}{\partial z}\right|-\alpha \frac{\partial \bar{p}_{0}}{\partial z} v_{\Gamma, 0}
$$

of a linear differential equation for the function $\bar{p}_{1}$. The solution exists if and only if the solvability condition holds

$$
-\kappa_{\Gamma, 0} \frac{\partial \bar{p}_{0}}{\partial z}-F\left(\bar{u}_{0}\right)\left|\frac{\partial \bar{p}_{0}}{\partial z}\right|-\alpha \frac{\partial \bar{p}_{0}}{\partial z} v_{\Gamma, 0} \perp \operatorname{Ker}\left(\frac{\partial^{2}}{\partial z^{2}}+f_{0}^{\prime}\left(\bar{p}_{0}\right)\right) .
$$

This implies that for each

$$
\begin{gathered}
\psi \in \operatorname{Ker}\left(\frac{\partial^{2}}{\partial z^{2}}+f_{0}^{\prime}\left(\bar{p}_{0}\right)\right) \\
\int_{\mathbb{R}}\left(-\kappa_{\Gamma, 0} \frac{\partial \bar{p}_{0}}{\partial z}-F\left(\bar{u}_{0}\right)\left|\frac{\partial \bar{p}_{0}}{\partial z}\right|-\alpha \frac{\partial \bar{p}_{0}}{\partial z} v_{\Gamma, 0}\right) \psi(z) \mathrm{d} z=0 .
\end{gathered}
$$

We observe that

$$
\frac{\partial \bar{p}_{0}}{\partial z} \in \operatorname{Ker}\left(\frac{\partial^{2}}{\partial z^{2}}+f_{0}^{\prime}\left(\bar{p}_{0}\right)\right)
$$

and we recover the Gibbs-Thompson relation on $\Gamma_{0}$

$$
\int_{\mathbb{R}}\left(-\kappa_{\Gamma, 0} \frac{\partial \bar{p}_{0}}{\partial z}-F\left(\bar{u}_{0}\right)\left|\frac{\partial \bar{p}_{0}}{\partial z}\right|-\alpha \frac{\partial \bar{p}_{0}}{\partial z} v_{\Gamma, 0}\right) \frac{\partial \bar{p}_{0}}{\partial z} \mathrm{~d} z=0 .
$$

We formally investigate the order of accuracy in relation (2.4) in terms of expansions in $\xi$. 
Lemma 4.6 The Gibbs-Thompson law on $\Gamma_{0}$ is satisfied up to order 2 in terms of formal asymptotic expansion:

$$
\begin{aligned}
\alpha v_{\Gamma, 0}+\kappa_{\Gamma, 0}-F\left(\bar{u}_{0}\right) & =0, \\
\alpha v_{\Gamma, 1}+\kappa_{\Gamma, 1}-F^{\prime}\left(\bar{u}_{0}\right) \bar{u}_{1} & =0 .
\end{aligned}
$$

Proof. We denote

$$
\mathcal{I}_{1}=\int_{\mathbb{R}} F\left(\bar{u}_{0}\right)\left|\frac{\partial \bar{p}_{0}}{\partial z}\right| \frac{\partial \bar{p}_{0}}{\partial z} \mathrm{~d} z, \quad \mathcal{I}_{2}=\int_{\mathbb{R}}\left(\frac{\partial \bar{p}_{0}}{\partial z}\right)^{2} \mathrm{~d} z .
$$

We rewrite relation (4.21) as

$$
\mathcal{I}_{2}\left(-\kappa_{\Gamma, 0}-\alpha v_{\Gamma, 0}\right)-\mathcal{I}_{1}=0,
$$

assuming that the quantities $\kappa_{\Gamma, 0}, v_{\Gamma, 0}$ do not depend on $z$ (obviously, they can vary along $s$ ). Consequently,

$$
\mathcal{I}_{2}=-\int_{\mathbb{R}} \sqrt{2 w_{0}\left(\bar{p}_{0}\right)} \frac{\partial \bar{p}_{0}}{\partial z} \mathrm{~d} z=\int_{0}^{1} \sqrt{2 w_{0}(p)} \mathrm{d} p,
$$

using properties of the double-well potential $w_{0}$ and the sign convention. The function $\bar{u}_{0}=$ const., from which finally

$$
\mathcal{I}_{1}=F\left(\bar{u}_{0}\right) \int_{\mathbb{R}}\left|\frac{\partial \bar{p}_{0}}{\partial z}\right| \frac{\partial \bar{p}_{0}}{\partial z} \mathrm{~d} z=-F\left(\bar{u}_{0}\right) \mathcal{I}_{2} .
$$

The Gibbs-Thompson condition at the $\mathcal{O}(1)$ level is then

$$
F\left(\bar{u}_{0}\right)=\kappa_{\Gamma, 0}+\alpha v_{\Gamma, 0} .
$$

To show (4.23), we recall that the relation (4.18) represents an equation for $\bar{p}_{2}$

$$
\frac{\partial^{2} \bar{p}_{2}}{\partial z^{2}}+f_{0}^{\prime}\left(\bar{p}_{0}\right) \bar{p}_{2}=G_{2},
$$

where using the properties of $\bar{u}_{0}, \bar{p}_{0}, \bar{p}_{1}$, and (4.22)

$$
\begin{aligned}
G_{2}= & -\frac{\partial \bar{p}_{0}}{\partial z}\left(\alpha v_{\Gamma, 1}+\kappa_{\Gamma, 1}+z \hat{\kappa}_{\Gamma, 0}\right)-\frac{\partial \bar{p}_{1}}{\partial z}\left(\alpha v_{\Gamma, 0}+\kappa_{\Gamma, 0}-F\left(\bar{u}_{0}\right)\right) \\
& -\frac{1}{2} f_{0}^{\prime \prime}\left(\bar{p}_{0}\right) \bar{p}_{1}^{2}-\left|\frac{\partial \bar{p}_{0}}{\partial z}\right| F^{\prime}\left(\bar{u}_{0}\right) \bar{u}_{1} .
\end{aligned}
$$

The solvability condition $G_{2} \perp \operatorname{Ker}\left(\frac{\partial^{2}}{\partial z^{2}}+f_{0}^{\prime}\left(\bar{p}_{0}\right)\right)$ and the fact that

$$
\int_{\mathbb{R}} f_{0}^{\prime \prime}\left(\bar{p}_{0}\right) \bar{p}_{1}{ }^{2} \frac{\partial \bar{p}_{0}}{\partial z} \mathrm{~d} z=0, \quad \int_{\mathbb{R}} z\left(\frac{\partial \bar{p}_{0}}{\partial z}\right)^{2} \mathrm{~d} z=0,
$$

imply

$$
\alpha v_{\Gamma, 1}+\kappa_{\Gamma, 1}-F^{\prime}\left(\bar{u}_{0}\right) \bar{u}_{1}=0 .
$$

Consequently, from (4.22) and (4.23), we see that relation (2.4) is satisfied by the solution of (2.5) up to order $\xi^{2}$. 
REMARK Relation (4.22) allows us to identify the parameters of (2.5) in order to achieve a quantitative agreement with the physical reality. Solving equation (4.19) in particular for $f_{0}(p)=$ ap $(1-p)\left(p-\frac{1}{2}\right)$ appearing in $(2.5)$, we get

$$
\bar{p}_{0}(z)=\frac{1}{2}\left(1-\tanh \left(\frac{z}{2} \sqrt{\frac{a}{2}}\right)\right),
$$

and

$$
\mathcal{I}_{2}=\frac{1}{6} \sqrt{\frac{a}{2}} .
$$

We also observe that the resulting relation (4.22) is independent on the quantity $\mathcal{I}_{2}$ (which can be cancelled on both sides of (4.21)), in contrast to the standard Allen-Cahn equation (see [16], [3]).

\section{Computational results}

Here, we present several numerical studies using the model (2.5) both with the coupling $\chi(p)=p$, and with non-trivial coupling

$$
\chi(p)=2^{2 r-1} p^{2 r} \quad \text { for } \quad p<\frac{1}{2}, \quad 1-2^{2 r-1}(1-p)^{2 r} \quad \text { for } \quad p \geqslant \frac{1}{2},
$$

mostly with $r=2$. We always set

$$
F(u)=\beta\left(u^{*}-u\right) \quad \text { for } \quad\left|u-u^{*}\right|<C_{u},
$$

and

$$
\begin{gathered}
F(u)=-C_{u} \beta \quad \text { for } \quad u \geqslant u^{*}+C_{u}, \\
F(u)=C_{u} \beta \quad \text { for } \quad u \leqslant u^{*}-C_{u},
\end{gathered}
$$

in order to satisfy basic theoretical requirements imposed on $F$. Here, $u^{*}$ denotes the melting point. If $C_{u}$ is sufficiently large, the model behaves as if $F$ were linear, and is applicable in real situations. The presented studies should clearly demonstrate the gain in accuracy with respect to the sharpinterface standards. On the other hand, they are certainly not exhausting and qualitatively complete. For details on this topic, see [3] and [5].

The numerical algorithm used to solve (2.5) has been described in [3] in detail. It is based on spatial discretization by finite differences on a uniform grid, and on the Runge-Kutta fourth-order time solver with adaptive time step.

The simplest benchmark test is to use the one-dimensional case, where there is an analytical solution to the Stefan problem with undercooling effects:

$$
\begin{aligned}
\frac{\partial u}{\partial t} & =\frac{\partial^{2} u}{\partial x^{2}} \quad \text { in } \Omega_{s} \subset \mathbb{R} \quad \text { and } \quad \Omega_{l} \subset \mathbb{R}, t>0, \\
\left.u\right|_{-\infty} & =u_{-\infty},\left.\quad u\right|_{+\infty}=u_{+\infty}, \\
\left.u\right|_{t=0} & =u_{\text {ini }}, \quad \text { for } \quad x \in \mathbb{R}, \\
\left.\frac{\partial u}{\partial n}\right|_{s}-\left.\frac{\partial u}{\partial n}\right|_{l} & =L v_{\Gamma} \quad \text { on } \quad \Gamma, \\
u-u^{*} & =-\frac{\alpha}{\beta} v_{\Gamma} \quad \text { on } \Gamma, \\
\left.\Omega_{S}(t)\right|_{t=0} & =\Omega_{s o} .
\end{aligned}
$$



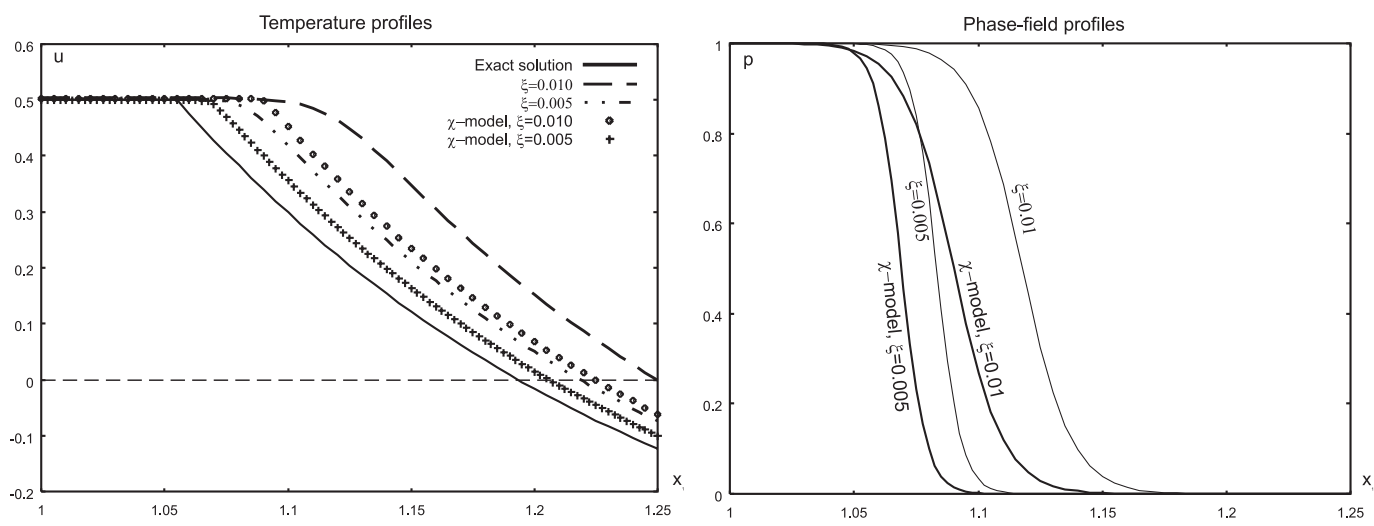

FIG. 2. One-dimensional travelling-wave solution of phase-field equations for the values of small parameter $\xi=0.01,0.005$ Other parameters are $u(t, 0)=0.5, u(t, 1)=-0.5, t>0, L=1.0, \beta=10.0, a=2.0, \alpha=1.0, u^{*}=0.0$, spatial domain of size $L_{1}=2.0$, spatial mesh sizes $h_{1}=0.005,0.0025$, respectively. The solutions for $\chi(p)=p$ and for non-trivial $\chi$ (denoted as $\chi$-model) are compared to the analytical solution of the Stefan problem with undercooling.
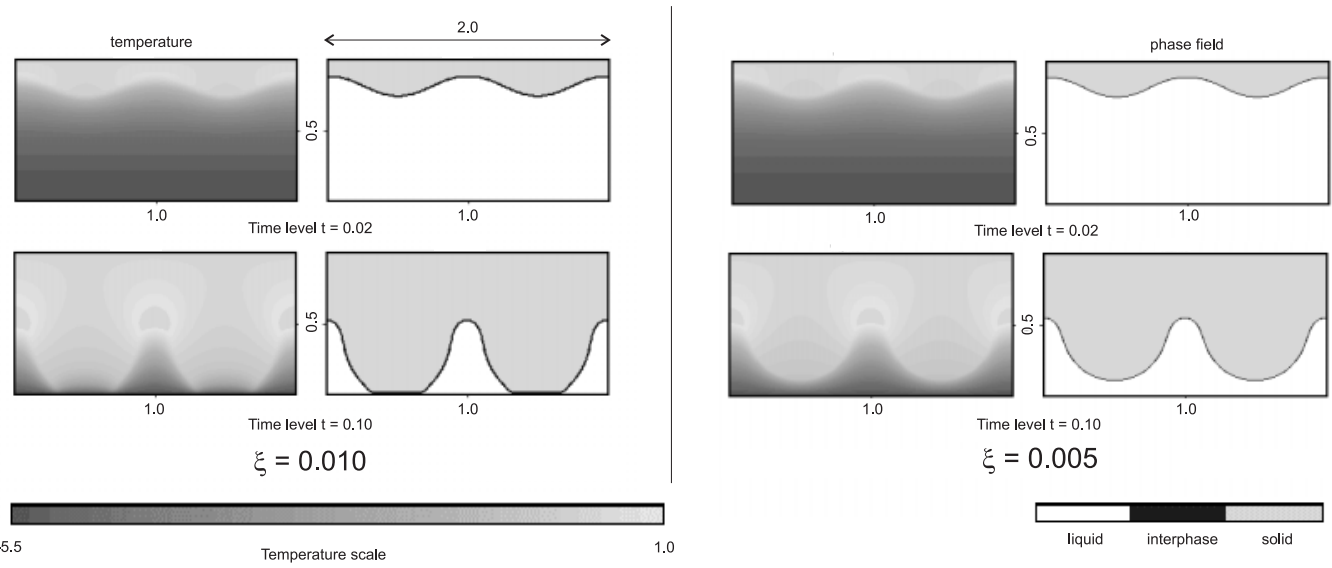

FIG. 3. Two-dimensional travelling-wave solution of phase-field equations with standard coupling $(\chi(p)=p)$ for the values of small parameter $\xi=0.01,0.005$. Temperature on the top edge is $u_{\text {top }}=1.0$, temperature on the bottom edge is $u_{\text {bottom }}=-5.5$, the side edges are adiabatically isolated. Other parameters are $L=6.0, \beta=10.0, a=2.0, \alpha=1.0$, $u^{*}=1.0$, the domain $\Omega=(0,2) \times(0,1)$, mesh $h_{1}=0.005,0.0025, h_{2}=0.005,0.0025$, respectively.

The solution of (5.1) has the form of a travelling wave

$$
\begin{aligned}
u(x, t)= & u_{-\infty} \quad \text { for } \quad x \leqslant v_{\Gamma} t, \\
& u_{-\infty}-L+L \exp \left(v_{\Gamma}\left(x-v_{\Gamma} t\right)\right) \quad \text { for } \quad x>v_{\Gamma} t,
\end{aligned}
$$

where

$$
u_{+\infty}=u_{-\infty}-L, v_{\Gamma}=\frac{\beta}{\alpha}\left(u^{*}-u\right)
$$

with $u_{-\infty}$ being the far-field temperature.

Figure 2 indicates the difference in convergence of the phase-field model (2.5) with standard and improved coupling $\chi$ towards the sharp-interface formulation (5.1). We observe a gain in distance to 

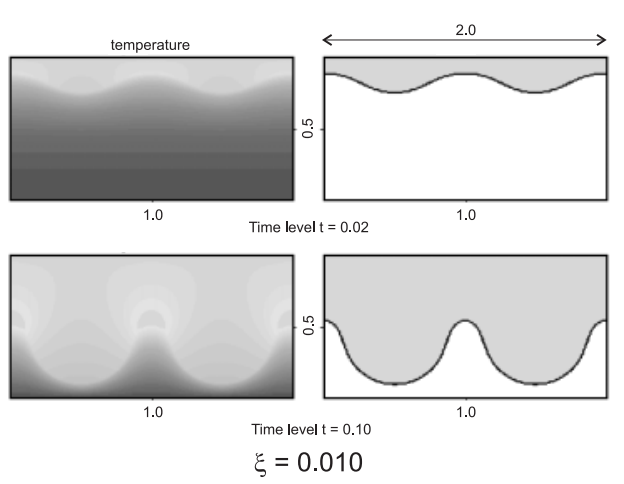

Temperature scale
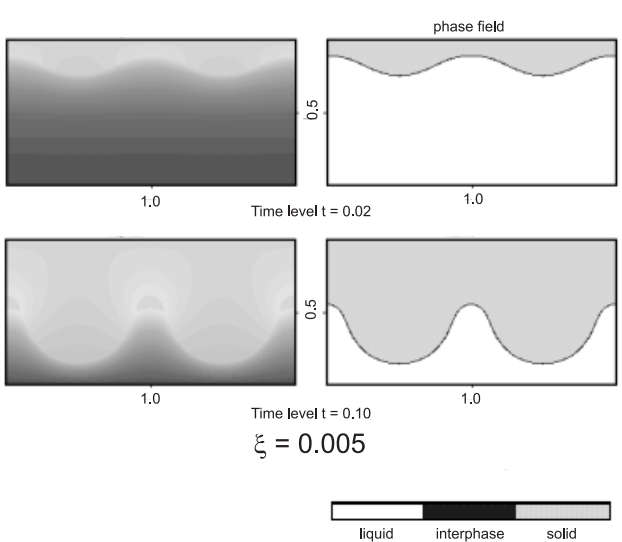

FIG. 4. Two-dimensional travelling-wave solution of phase-field equations with improved coupling $\left(\chi^{\prime}(s)=r 2^{2 r} s^{2 r-1}\right.$ for $s<0.5, \chi^{\prime}(s)=r 2^{2 r}(1-s)^{2 r-1}$ for $s \geqslant 0.5$ with $\left.r=2\right)$ for the values of small parameter $\xi=0.01,0.005$. Temperature on the top edge is $u_{\text {top }}=1.0$, temperature on the bottom edge is $u_{\text {bottom }}=-5.5$, the side edges are adiabatically isolated. Other parameters are $L=6.0, \beta=10.0, a=2.0, \alpha=1.0, u^{*}=1.0$, the domain $\Omega=(0,2) \times(0,1)$, mesh $h_{1}=0.005,0.0025$, $h_{2}=0.005,0.0025$, respectively.

the analytical solution, and also in the shape of the numerically obtained temperature field. Figures 3 and 4 show the difference in the solution of the same two-dimensional problem if changing $\xi$. The equations with non-trivial coupling $\chi$ exhibit more stable behaviour with respect to $\xi$.

\section{Acknowledgements}

The author was partly supported by project MSM J04/98/210000010 of the Ministry of Education of the Czech Republic: and by the project no. 201/01/0676 of the Grant Agency of the Czech Republic. The support of the Laboratoire de Métalurgie Physique, Département des Matériaux, École Polytechnique Fédérale de Lausanne concerning computational tools is gratefully acknowledged.

\section{REFERENCES}

1. Alikakos, N. D. \& Bates, P. W. On the singular limit in a phase field model of phase transitions. Ann. Inst. Henri Poincaré 5, (1988) 141-178.

2. BENEŠ, M. On a computational comparison of phase-field and sharp-interface model of microstructure growth in solidification. Acta Techn. CSAV 41, (1996) 597-608.

3. BENEŠ, M. Phase-field model of microstructure growth in solidification of pure substances, $\mathrm{PhD}$ Thesis, Faculty of Nuclear Sciences and Physical Engineering, Czech Technical University, (1997).

4. Beneš, M. \& Mikula, K. Simulation of anisotropic motion by mean curvature-comparison of phasefield and sharp-interface approaches. Acta Math. Univ. Comenianae 67, (1998) 17-42.

5. BENEŠ, M. Numerical simulation of microstructure growth in solidification by the phase-field model with a gradient coupling term. In: VAn KeER, R., Verhegghe, R., Hogge, M. \& Noldus, E. (eds), ACOMEN'98, Advanced Computational Methods in Engineering. pp. 343-350. Maastricht (1998).

6. BRONSARD, L. \& KOHN, R. Motion by mean curvature as the singular limit of Ginzburg-Landau dynamics. J. Diff. Eqns. 90, (1991) 211-237. 
7. Caginalp, G. An analysis of a phase field model of a free boundary. Arch. Rational Mech. Anal. 92, (1986) 205-245.

8. Caginalp, G. Stefan and Hele-Shaw type models as asymptotic limits of the phase-field equations. Phys. Rev. A 39, (1989) 5887-5896.

9. CAhn, J. W. \& Hilliard, J. E. Free energy of a nonuniform system. I. interfacial free energy. J. Chem. Phys. 28, (1958) 258-267.

10. Fierro, F. \& PaOlini, M. Numerical evidence of fattening for the mean curvature flow. Math. Models Methods Appl. Sci. 6, (1996) 793-813.

11. GiUsti, E. Minimal Surfaces and Functions of Bounded Variation. Birkha̋user, Basel (1984).

12. Grisvard, P. Elliptic Problems in Nonsmooth Domains. Pitman, Boston (1985).

13. Gurtin, M. Thermomechanics of Evolving Phase Boundaries in the Plane. Clarendon, Oxford (1993).

14. LAdYZHENSKAYA, O. A. Mathematical Aspects of Dynamics of Viscous Incompressible Fluids. Nauka, Moscow (1970) In Russian.

15. Lagerstrom, P. A. Matched Asymtotic Expansions. Springer, New York (1988).

16. PAOLINI, M. \& Verdi, C. Asymptotic and numerical analyses of the mean curvature flow with a spacedependent relaxation parameter. Asymptotic Anal. 5, (1992) 553-574.

17. RoubíčEK, T. A generalization of the Lions-Temam compact imbedding theorem. Čas. Pěst. Matem. 115, (1990) 338-342.

18. Temam, R. Navier-Stokes Equations, Theory and Numerical Analysis. North-Holland, Amsterdam (1979). 